\title{
Parameters tuning of power system stabilizers using improved ant direction hybrid differential evolution
}

\author{
Sheng-Kuan Wang ${ }^{\mathrm{a}, \mathrm{b}, *}$, Ji-Pyng Chiou ${ }^{\mathrm{b}}$, Chih-Wen Liu ${ }^{\mathrm{a}}$ \\ a Electrical Engineering, National Taiwan University, No. 1, Sec. 4, Roosevelt Road, Taipei 10617, Taiwan, ROC \\ ${ }^{\mathrm{b}}$ Electrical Engineering, Ming Chi University of Technology, 84 Gungjuan Rd., Taishan, Taipei 24301, Taiwan, ROC
}

\section{A R T I C L E I N F O}

\section{Article history:}

Received 15 February 2008

Received in revised form 17 September

2008

Accepted 18 October 2008

\section{Keywords:}

Hybrid differential evolution

Power system stabilizer

Dynamic stability

Optimization

Eigenvalue analysis

\begin{abstract}
A B S T R A C T
The tuning of the PSS parameters for a multi-machine power system is usually formulated as an objective function with constraints consisting of the damping factor and damping ratio. A novel mixed-integer ant direction hybrid differential evolution algorithm, called MIADHDE, is proposed to solve this kind of problem. The MIADHDE is improved from ADHDE by the addition of accelerated phase and real variables. The performances of three different objective functions are compared to the MIADHDE in this paper. Both local and remote feedback signals of machine speed deviation measurements can be selected as input signals to the PSS controllers in the proposed objective function. The New England 10-unit 39-bus standard power system, under various system configurations and loading conditions, is employed to illustrate the performance of the proposed method with the three different objective functions. Eigenvalue analysis and nonlinear time domain simulation results demonstrate the effectiveness of the proposed algorithm and the objective function with a remote signal.
\end{abstract}

(c) 2008 Elsevier Ltd. All rights reserved.

\section{Introduction}

The dynamic stability of power systems is an important factor for secure system operation. Low-frequency oscillation modes have been observed when power systems are interconnected by weak tie lines $[1,2]$. The low-frequency oscillation mode, which has poor damping in a power system, is also called the electromechanical oscillation mode and usually occurs in the frequency range of $0.1-2.0 \mathrm{~Hz}$ [3-6]. The power system stabilizer (PSS) has been widely used for mitigating the effects of low-frequency oscillation modes [7]. The construct and parameters of PSS have been discussed in many studies [3-6]. Currently, many plants prefer to employ conventional lead-lag structure PSSs, due to the ease of online tuning and reliability [7-9]. Over the last two decades, various parameter tuning schemes of PSS have been developed and applied to solve the problem of dynamic instability in a power system. The parameter tuning of PSSs in the power system has two major methods, sequential tuning and simultaneous tuning [8]. In order to obtain the set of optimal PSS parameters under various operating conditions, the tuning and testing of PSS parameters must be repeated under various system operating conditions. Therefore, if the sequential tuning method is applied to tuning PSS parameters,

\footnotetext{
* Corresponding author. Address: Electrical Engineering, Ming Chi University of Technology, 84 Gungjuan Rd., Taishan, Taipei 24301, Taiwan, ROC. Tel.: +886 2 29089899; fax: +886229084507.

E-mail address: d90921002@ntu.edu.tw (S.-K. Wang).
}

the parameters tuning will become more complicated, and the tuned result maybe not a locally or globally optimal solution. On the other hand, in the case that the simultaneous tuning method is employed in tuning of PSS parameters, which can simultaneously relocate and coordinate the eigenvalues of various oscillation modes under different operating conditions, the set of PSS parameters solutions can quite close to the globally optimal solution. However, the drawback of the simultaneous tuning method is the longer computation time required for large power systems. The simultaneous tuning of PSS parameters is usually formulated as a very large scale nonlinear non-differentiable optimization problem. This kind of optimization problem is very hard, if not impossible, to solve using traditionally differentiable optimization algorithms. Instead, we propose a stochastic optimization approach.

Abdel-Magid and Abido [8,10-13] have employed the tabu search (TS), simulated annealing (SA), particle swarm optimization (PSO), evolutionary programming (EP), and genetic algorithm (GA) to optimize the parameters of the PSSs in the New England ten-machine system. In the system, all PSSs are simultaneously designed to take into account mutual interactions. The objective function is devised to optimize the desired damping factor $(\sigma)$ and/or the desired damping ratio $(\xi)$ of the lightly damped and undamped modes. In this way, only the unstable or lightly damped oscillation modes are relocated. Hongesombut et al. [9] used hierarchical and parallel micro genetic algorithms in a multi-machine system. Do Bomfim et al. [14] used genetic algorithms to simultaneously tune 


\begin{tabular}{|c|c|}
\hline \multicolumn{2}{|l|}{ Nomenclature } \\
\hline General & constant coefficient \\
\hline damping factor & probability of choosing a mutation operator \\
\hline$\Delta \omega \quad$ speed deviation & influence factor of heuristic information \\
\hline$T_{1}, T_{2}, T_{3}, T_{4}$ lead/lag time constants of PSS & perturbed individual vector of $i^{\text {th }}$ individual for integer \\
\hline$n \quad$ amount & variables \\
\hline weight constant & $C_{r} \quad$ crossover factor \\
\hline real variables & arg min the argument of the minimum \\
\hline random number & $\varepsilon_{2} \quad$ desired tolerance for the gene diversity \\
\hline $\begin{array}{ll}\tau_{i} & \text { pheromone information } \\
\text { object } & \text { objective function value }\end{array}$ & $\bar{\alpha} \quad$ step size \\
\hline$\eta_{i} \quad$ heuristic information & Subscripts \\
\hline influence factor of pheromone information & $Z=1,2, \ldots, n_{Z}$ index of local machines \\
\hline $\begin{array}{l}\text { difference vector between individual } \mathrm{j} \text { and } \mathrm{k} \text { for integer } \\
\text { variables }\end{array}$ & $R Z=0,1, \ldots, n_{Z}$ index of remote machines \\
\hline scaling factor & \\
\hline $\begin{array}{l}h^{\text {th }} \text { gene of } i^{\text {th }} \text { trial individual vector of }(G+1)^{\text {th }} \text { genera- } \\
\text { tion }\end{array}$ & $\begin{array}{l}h=1, \ldots, n \text { index of integer variable } \\
N \quad N^{\text {th }} \text { PSS }\end{array}$ \\
\hline$\varepsilon_{1} \quad$ desired tolerance for the population diversity & \\
\hline$\left(\bar{I}_{b}^{G+1}, \bar{X}_{b}^{G+1}\right)$ best individual & $y=1,2, \ldots, n_{y} \quad$ index of system operating conditions \\
\hline$\xi^{D} \quad{ }^{D}$ damping ratio & $i=1,2, \ldots, N_{P}$ index of individuals \\
\hline rotor angle & $j \quad$ index of the genes \\
\hline gain of the PSS & \multirow[t]{2}{*}{$g=(n+1), \ldots, m$ index of real variable } \\
\hline expected damping factor & \\
\hline expected damping ratio & Superscripts \\
\hline integer variables & $0 \quad$ initial value \\
\hline number of individuals & index of the generation \\
\hline proportion constant of pheromone & best value \\
\hline
\end{tabular}

multiple power system damping controllers with the objective function of the sum of the spectrum damping ratios for all operating conditions. These studies did not consider the remote feedback signals, which are available from synchronized phasor measurement units [15-17]. Kamwa et al. [18,19] used a decentralized/ hierarchical control system with two global signals and one local signal as input of PSSs. The global signals obtained the highest controllability of these oscillation modes. Hasanović and Feliachi [20] used a genetic algorithm to accomplish simultaneous tuning of multiple power system damping controllers. Both local and remote measurement signals have been considered as input signals to the damping controllers. However, the remote measurements are considered only in a 4-machine system, and that remote signal is not determined from the computational results. Su and Lee $[21,22]$ used improved mixed-integer hybrid differential evolution to solving network reconfiguration and capacitor placement problems of a distribution system, but those problems only contain integer decision variables.

Chiou et al. [23] developed the ant direction hybrid differential evolution (ADHDE) method, which utilizes the concept of an ant colony search to find a suitable mutation strategy in the HDE method $[24,25]$ to accelerate the search for the global solution. The ADHDE has proved that it can obtain excellent computation results to the optimization problems of integer variables in the capacitor placement problem [23], but it has not been investigated in terms optimization problems consisting of both integer and real variables. In order to rapidly find a global solution by ADHDE, the accelerated phase is added in this algorithm. In this study, the machine numbers of remote feedback signals are regarded as the integer variable. Therefore, the proposed optimization algorithm must have the ability to search out the set of optimal solutions of integer and real variables at the same time. The optimization problems of both real and integer variables are generally called mixed-integer nonlinear programming problems. The original ADHDE algorithm gives it the ability to search out a set optimal solution of integer and real variables at the same time, so the algorithm is called the mixed-integer ant direction hybrid differential evolution algorithm (MIADHDE). The MIADHDE algorithm in this study is proposed to determine the optimal gain, time constants, and machine numbers of remote feedback signals of PSSs for the multi-machine system by three different objective functions. The three objective functions will be compared in terms of performance by the degree of system damped.

\section{Problem formulation}

\subsection{Power system model}

In this study, each generator is modeled as a two-axis model, which is a six-order model. The state vector of the generator is given as $\left[\Delta \omega, \delta, \phi_{f d}, \phi_{1 d}, \phi_{1 q}, \phi_{2 q}\right]$, where $\Delta \omega$ and $\delta$ are the speed deviation and rotor angle, respectively, and $\phi_{f d}, \phi_{1 d}, \phi_{1 q}$ and $\phi_{2 q}$ are the contribution to the rotor flux linkage as a result of field winding, one $d$-axis and two $q$-axis amortisseur circuits, respectively [26]. These device models of a power system include generators, PSSs, and excitation systems, and can be formulated by:

$\dot{x}=f(x, r, t)$

where $x$ is the vector of all state variables, and $r$ is the vector of input variables. In the PSS design, the power system is usually linearized in terms of a perturbed value in order to perform the small signal analysis. Therefore, Eq. (1) can be represented as:

$\left\{\begin{array}{l}\Delta \dot{x}=A \Delta x+B \Delta v \\ \Delta u=C \Delta x+D \Delta v\end{array}\right.$

where $A$ is the power system state matrix, $B$ is the input matrix, $C$ is the output matrix, $D$ is the feedforward matrix, $v$ is the vector of the 
network bus voltages, and $u$ is the current vector of the injection into the network from the device [26].

The PSSs with a lead-lag structure of speed deviation input are considered in this study, and the transfer function of the $N^{\text {th }}$ PSS is given by Eq. (3). It contains a remote feedback signal of speed deviation, $\Delta \omega_{R Z}$.

$V_{S N}(s)=K_{S N} \frac{s T_{W N}}{\left(1+s T_{W N}\right)} \frac{\left(1+s T_{1 N}\right)}{\left(1+s T_{2 N}\right)} \frac{\left(1+s T_{3 N}\right)}{\left(1+s T_{4 N}\right)}\left(\Delta \omega_{Z}-\Delta \omega_{R Z}\right)$

where $T_{1 N}, T_{2 N}, T_{3 N}, T_{4 N}$, and $K_{S N}$ are the time constants and gain of the $N^{\text {th }}$ PSS, $Z=1,2, \ldots, n_{Z}$ is the index of machines, $n_{Z}$ is the number of machines, $\Delta \omega_{Z}$ is the speed deviation of the $Z^{\text {th }}$ local machine, $\Delta \omega_{R Z}$ is the speed deviation of the $R Z^{\text {th }}$ remote machine, $R Z=0,1$, $2, \ldots, n_{Z}$ is the index of remote machines, and $R Z=0$ represents that the PSS has no remote feedback signal, $\Delta \omega_{R Z}=0$. However, the conservative assumption that the effect of communication delay can be neglected is made. The time constant $T_{W N}$ in the washout filter is usually kept constant during the optimization. Which typical value is between 10 and 20 so that obtains well phase compensation performance. However, the washout time constant is fixed at 10 in this study.

\subsection{Objective function}

When a disturbance occurs, the decaying rate of the power system oscillation is dominated by the maximum damping factor in the system, and the amplitude of the each oscillation mode is determined by its damping ratio. Thus, the objective functions naturally include the damping factor and the damping ratio in the formulation for the tuning of PSS parameters. In this paper, three objective functions are considered.

(1) The first objective function for the optimal PSS parameters may be formulated as follows:

$\operatorname{Min} F_{1}=\sum_{y=1}^{n_{y}} \sum_{\sigma_{q, y} \geqslant \sigma_{0}}\left(\sigma_{q, y}-\sigma_{0}\right)^{2}+\varpi \cdot \sum_{y=1}^{n_{y}} \sum_{\zeta_{q, y} \leqslant \zeta_{0}}\left(\zeta_{0}-\zeta_{q, y}\right)$

subject to

$\left\{\begin{array}{l}K_{S N, \text { min }} \leqslant K_{S N} \leqslant K_{S N, \text { max }} \\ T_{1 N, \text { min }} \leqslant T_{1 N} \leqslant T_{1 N, \text { max }} \\ T_{2 N, \text { min }} \leqslant T_{2 N} \leqslant T_{2 N, \text { max }} \\ T_{3 N, \text { min }} \leqslant T_{3 N} \leqslant T_{3 N, \text { max }} \\ T_{4 N, \text { min }} \leqslant T_{4 N} \leqslant T_{4 N, \text { max }}\end{array}\right.$

where $y=1,2,3, \ldots, n_{y}$ is the index of system operating conditions considered in this design process, $q=1,2, \ldots, n_{q}$, is the index of eigenvalues in the system, $\sigma_{q . y}$ is the damping factor of the $q^{\text {th }}$ eigenvalue of the $y^{\text {th }}$ operating condition, $\sigma_{0}$ is a constant value of the expected damping factor, $\varpi$ is a weight for combining both damping factors and damping ratios, $\zeta_{q . y}$ is the damping ratio of the $q^{\text {th }}$ eigenvalue of the $y^{\text {th }}$ operating condition, and finally, $\zeta_{0}$ is a constant value of the expected damping ratio. The constraint set is made up of bounds of PSS parameters, which can be formulated as Eq. (5), and the speed deviation measurement signals of the remote machine are neglected; $\Delta \omega_{R Z}=0$ in this formulation. The objective function only forces the unstable or lightly damped electromechanical oscillation modes to be relocated, an objective function similar to the best one in the literature [8]. In the Eq. (4), the damping ratio difference is less than 1 ; if we take each square for this term, then the objective function is unable to determine the optimum solution. The best objective value is zero for this function.

(2) The second objective function for tuning PSS parameters may be chosen to minimize the following function.
$\operatorname{Min} F_{2}=\sum_{y=1}^{n_{y}}\left(\max _{1 \leqslant q \leqslant n_{q}} \sigma_{q}-\sigma_{0}\right)_{y}+\varpi \sum_{y=1}^{n_{y}}\left(\zeta_{0}-\min _{1 \leqslant q \leqslant n_{q}} \zeta_{q}\right)_{y}$

where $\max \sigma_{q}$ and $\min \zeta_{q}$ are the maximum real part of the eigenvalues and the minimum of the damping ratio, respectively, in the $y^{\text {th }}$ system operating condition. The objective function not only relocates the unstable or lightly damped oscillation modes to the left side of the s-plane but also shifts other oscillation modes more to the left side of the s-plane. The same constraints as Eq. (5) are used, and the speed deviation measurement signals of the remote machine are neglected, so $\Delta \omega_{R Z}=0$ in this formulation.

3) The third objective function is the same as Eq. (6) but has different constraints, as follows.

$\operatorname{Min} F_{3}=\sum_{y=1}^{n_{y}}\left(\max _{1 \leqslant q \leqslant n_{q}} \sigma_{q}-\sigma_{0}\right)_{y}+\varpi \sum_{y=1}^{n_{y}}\left(\zeta_{0}-\min _{1 \leqslant q \leqslant n_{q}} \zeta_{q}\right)_{y}$

The constraints add the speed deviation measurement signals of remote machines for the input to PSSs in Eq. (8), except the same constraints as Eq. (5) are used.

$0 \leqslant R Z \leqslant n_{Z}$, where $R Z \neq Z$

\section{MIADHDE algorithm}

The ADHDE algorithm developed by Chiou et al. can be found in the literature [23]. The main idea of ADHDE is to use the ant colony search system to find the proper mutation operator to accelerate the search for the global solution. The MIADHDE algorithm improves the ADHDE to deal with the optimization problem of simultaneously including integer and real variables and to add the accelerated phase with the aim of faster convergence. The MIADHDE algorithm is briefly described below.

Step 1. Initialization

The initial population and load system data are created. Some initial populations are randomly chosen, and they endeavor to cover the complete parameter space uniformly. The elements of every random variable $\left(I_{i}^{0}, X_{i}^{0}\right)$ are assumed as follows

$$
\begin{gathered}
\left(I_{i}^{0}, X_{i}^{0}\right)=\left(I_{i, \min }, X_{i, \min }\right)+\left(I N T\left(\vartheta_{i}\left(I_{i, \max }-I_{i, \min }\right)\right),\right. \\
\left.\vartheta_{i}\left(X_{i, \max }-X_{i, \min }\right)\right), \quad i=1, \ldots, N_{p}
\end{gathered}
$$

where $\vartheta_{i} \in(0,1]$ is a random number, and INT operator is represented to take the nearest integer. The two variables, $X$ and $I$, are real and integer variables, respectively. The initial process can create $N_{p}$ individuals of $\left(I_{i}^{0}, X_{i}^{0}\right)$ randomly.

Step 2. Ant direction search

In every generation, each of $N_{p}$ ants selects a mutation operator according to heuristic information and pheromone information. Different from [27-32], the difference between the objective function value in the next generation and the best objective function value of the present generation constructs a fluctuant pheromone quantity. The fluctuant pheromone quantity is defined as follows:

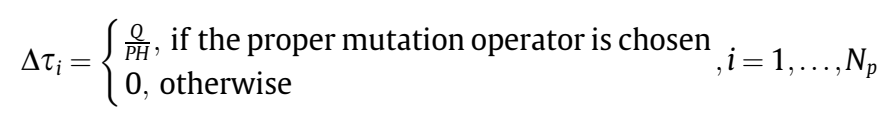

In Eq. (10), the proper mutation operator is chosen when the objective function value of the next generation is better than the best objective function value of the present generation. Where $Q$ is a constant, $P H$ is defined as follows:

$P H=\left|\frac{\text { object }_{\text {new }}}{\text { object }_{\text {prest }}^{\text {pest }}-\text { object }_{\text {new }}}\right|$ 
where object $t_{\text {present }}^{\text {best }}$ expresses the best objective function value of the present generation and object $_{n e w}$ expresses the objective function value of the next generation. The pheromone updating applies the following rule.

$\tau_{i}^{\text {New }}=\rho \cdot \tau_{i}^{\text {old }}+\Delta \tau_{i}$

The coefficient $\rho$ must be fixed to a value $<1$ to avoid an unlimited accumulation of trace. The magnitude of the new pheromone is updated by vaporizing $(1-\rho)$ percent of the pheromones from the previous iteration and the fluctuant pheromone quantity in the current iteration. It is observed that the probability of selecting a mutation operator is proportional to the pheromone quantity $\tau_{i}$ and the information $\eta_{i}$.. The information $\eta_{i}$ is defined as follows:

$\eta_{i}=\sqrt{\sum_{j=1}^{n}\left(\left(I_{i j}^{G}-I_{b j}^{G}\right) / I_{i j}^{G}\right)^{2}+\sum_{j=n+1}^{m}\left(\left(X_{i j}^{G}-X_{b j}^{G}\right) / X_{i j}^{G}\right)^{2}}$

where $\left(I_{i j}^{G}, X_{i j}^{G}\right)$ denotes the $j^{\text {th }}$ gene of the $i^{\text {th }}$ individual in a population in the $G^{\text {th }}$ generation, and $\left(I_{b j}^{G}, X_{b j}^{G}\right)$ denotes the $j^{\text {th }}$ gene of the best individual in a population in the $G^{\text {th }}$ generation. Hence, the probability of choosing a mutation operator is defined as follows:

$p_{i}(t)=\frac{\tau_{k}^{\alpha}(t) \cdot \eta_{k}^{\beta}}{\sum_{k=1}^{N_{p}} \tau_{k}^{\alpha}(t) \cdot \eta_{k}^{\beta}}$

where $\alpha$ and $\beta$ are parameters to regulate the influence of $\tau_{i}$ and $\eta_{i}$, respectively. At the same time, the probabilities are arranged in ascending order. The initial value of probability for choosing a mutation operator is first defined as $P=\left[p_{1}, p_{2}, p_{3}, p_{4}, p_{5}\right]=[0.2$, $0.4,0.6,0.8,1.0]$. Subsequently, the five integers $1,2,3,4,5$ are randomly arranged. For example, if the resultant arrangement is $[1,3$, $5,2,4]$, then by relating this arrangement to $P$, we have the probabilities of choosing mutation operators for strategy $1=0 \sim 0.2$, strategy $3=0.2 \sim 0.4$, strategy $5=0.4 \sim 0.6$, strategy $2=0.6 \sim 0.8$, and strategy $4=0.8 \sim 1.0$.

Step 3. Mutation operation

According to the probability of choosing a mutation strategy, the corresponding mutation operation and a random number $\chi \in[0,1]$ determine the proper mutation strategy. Assume that the probability of choosing a mutation operator is $P=[0.313$, $0.641,0.647,0.813,0.981]$, and the corresponding mutation operation is $[1,3,5,2,4]$. Suppose that the random number $\chi$ is equal to 0.567 , which is between 0.313 and 0.641 ; then the third mutation strategy is determined. This process is repeated for every individual.

Five mutation strategies have been introduced by [33-34]. The essential ingredient in the mutation operation is the difference vector. Each individual pair in a population at the $G^{\text {th }}$ generation defines a difference vector $\left(\tilde{I}_{j k}, \tilde{X}_{j k}\right)$ as

$\left\{\begin{array}{l}\tilde{I}_{j k}=I_{j}^{G}-I_{k}^{G} \\ \tilde{X}_{j k}=X_{j}^{G}-X_{k}^{G}\end{array}\right.$

The mutation process at the $G^{\text {th }}$ generation begins by randomly selecting either two or four population individuals $\left(I_{j}^{G}, X_{j}^{G}\right),\left(I_{k}^{G}, X_{k}^{G}\right),\left(I_{l}^{G}, X_{l}^{G}\right)$ and $\left(I_{m}^{G}, X_{m}^{G}\right)$ for any $j, k$, $l$ and $m$, according to five mutation strategies. These four individuals are then combined to form a difference vector $\left(\tilde{I}_{j k l m}, \tilde{X}_{j k l m}\right)$ as

$$
\left\{\begin{array}{l}
\tilde{I}_{j k l m}=\tilde{I}_{j k}+\tilde{I}_{l m}=\left(I_{j}^{G}-I_{k}^{G}\right)+\left(I_{l}^{G}-I_{m}^{G}\right) \\
\tilde{X}_{j k l m}=\tilde{X}_{j k}+\tilde{X}_{l m}=\left(X_{j}^{G}-X_{k}^{G}\right)+\left(X_{l}^{G}-X_{m}^{G}\right)
\end{array}\right.
$$

A perturbed individual is then generated based on the present individual in the mutation process by

$\left(\hat{I}_{i}^{G+1}, \hat{X}_{i}^{G+1}\right)=\left(I_{p}^{G}, X_{p}^{G}\right)+\left(I N T\left(\kappa \cdot \tilde{I}_{j k l m}\right), \kappa \cdot \tilde{X}_{j k l m}\right)$ where the scaling factor, $\kappa$, is a constant, and $j, k, l$ and $m$ are randomly selected.

The perturbed individual in Eq. (17) is essentially a noisy replica of $\left(I_{p}^{G}, X_{p}^{G}\right)$. Herein, the parent individual $\left(I_{p}^{G}, X_{p}^{G}\right)$ depends on the circumstance in which the type of the mutation operations is employed.

Step 4. Crossover operation

In order to extend the diversity of further individuals at the next generation, the perturbed individual of $\left(\hat{X}_{i}^{G+1}, \hat{I}_{i}^{G+1}\right)$ and the present individual of $\left(X_{i}^{G}, I_{i}^{G}\right)$ are chosen by a binomial distribution to progress the crossover operation to generate the offspring. Each gene of the $i^{\text {th }}$ individual is reproduced from the perturbed individual $\left(\hat{I}_{i}^{G+1}, \hat{X}_{i}^{G+1}\right)=\left[\hat{I}_{i 1}^{G+1}, \hat{I}_{i 2}^{G+1}, \ldots, \hat{I}_{i n}^{G+1}, \quad \hat{X}_{i(n+1)}^{G+1}, \hat{X}_{i(n+2)}^{G+1}, \ldots, \hat{X}_{i m}^{G+1}\right]$ and the present individual $\left(I_{i}^{G}, X_{i}^{G}\right)=\left[I_{i 1}^{G}, I_{i 2}^{G}, \ldots, I_{i n}, X_{i(n+1)}^{G}, X_{i(n+2)}^{G}, \ldots, X_{i m}^{G},\right]$. That is,

$$
\left\{\begin{array}{l}
\breve{I}_{i h}^{G+1}=\left\{\begin{array}{l}
I_{i h}^{G}, \text { if a random number }>C_{r} \\
\hat{I}_{i h}^{G+1}, \text { otherwise }
\end{array}\right. \\
\breve{X}_{i g}^{G+1}=\left\{\begin{array}{l}
X_{i g}^{G}, \text { if a random number }>C_{r} \\
\hat{X}_{i g}^{G+1}, \text { otherwise }
\end{array}\right.
\end{array}\right.
$$

where $i=1, \ldots, N_{p} ; h=1, \ldots, n ; g=(n+1), \ldots, m$, and the crossover factor $C_{r} \in[0,1]$ is assigned by the user.

Step 5. Estimation and selection

The evaluation function of a child is one-to-one competed to that of its parent. This competition means that the parent is replaced by its child if the fitness of the child is better than that of its parent. On the other hand, the parent is retained in the next generation if the fitness of the child is worse than that of its parent, i.e.

$\left(I_{i}^{G+1}, X_{i}^{G+1}\right)=\arg \min \left\{F\left(I_{i}^{G}, X_{i}^{G}\right), F\left(\breve{I}_{i}^{G+1}, \breve{X}_{i}^{G+1}\right)\right\}$

$\left(I_{b}^{G+1}, X_{b}^{G+1}\right)=\arg \min \left\{F\left(I_{i}^{G+1}, X_{i}^{G+1}\right)\right\}$

where arg min means the argument of the minimum.

Step 6. Migrating operation if necessary

In order to effectively enhance the investigation of the search space and reduce the choice pressure of a small population, a migration phase is introduced to regenerate a new diverse population of individuals. The best individual $\left(I_{b}^{G+1}, X_{b}^{G+1}\right)$ is selected to create the new population. The $h^{\text {th }}$ or $g^{\text {th }}$ gene of the $i^{\text {th }}$ individual is as in equation Eq. (21) and where $\vartheta_{i h}, \vartheta_{\text {ig }}$ and $\lambda$ are randomly generated numbers uniformly distributed in the range of $[0,1]$

$$
\left\{\begin{array}{l}
I_{i h}^{G+1}=\left\{\begin{array}{l}
I_{b h}^{G+1}+I N T\left(\vartheta_{i h} \cdot\left(I_{h \min }-I_{b h}^{G+1}\right)\right), \text { if } \lambda<\frac{I_{b h}^{G+1}-I_{h \min }}{I_{h \max }-I_{h \min }} \\
I_{b h}^{G+1}+I N T\left(\vartheta_{i h} \cdot\left(I_{h \max }-I_{b h}^{G+1}\right)\right), \text { otherwise }
\end{array}\right. \\
X_{i g}^{G+1}=\left\{\begin{array}{l}
X_{b g}^{G+1}+\vartheta_{i g} \cdot\left(X_{g \min }-X_{b g}^{G+1}\right), \text { if } \lambda<\frac{X_{b g}^{G+1}-X_{g \min }}{X_{g \max }-X_{g \min }} \\
X_{b g}^{G+1}+\vartheta_{i g} \cdot\left(X_{g \max }-X_{b g}^{G+1}\right), \text { otherwise }
\end{array}\right.
\end{array}\right.
$$

The migrating operation is executed only if a measure fails to match the desired tolerance of population diversity. The measure is defined as Eq. (22).

$\varepsilon=\sum_{\substack{i=1 \\ i \neq b}}^{N_{p}}\left(\sum_{j=1}^{n} \eta_{I}+\sum_{j=n+1}^{m} \eta_{X}\right) /\left(m \cdot\left(N_{p}-1\right)\right)<\varepsilon_{1}$

where

$$
\left\{\begin{array}{l}
\eta_{I}= \begin{cases}0, & \text { if } I_{i j}^{G+1}=I_{i b}^{G+1} \\
1, & \text { otherwise }\end{cases} \\
\eta_{X}= \begin{cases}0, & \text { if }\left|\frac{X_{i j}^{G+1}-X_{i b}^{G+1}}{X_{i b}^{G+1}}\right|<\varepsilon_{2} \\
1, & \text { otherwise }\end{cases}
\end{array}\right.
$$


Parameters $\varepsilon_{1}, \varepsilon_{2} \in[0,1]$ express the desired tolerance for the population diversity and the gene diversity with respect to the best individual, and $\eta_{I}$ and $\eta_{X}$ are the scale indices. From Eqs. (22) and (23), it can be seen that the value $\varepsilon$ is in the range of $[0,1]$. If $\varepsilon$ is smaller than $\varepsilon_{1}$, then the migrating operation is executed to generate a new population to escape the local point; otherwise, the migrating operation is turned off.

Step 7. Accelerated operation if necessary

When the best individual at the present generation is not improved any longer by the mutation and crossover operations, a decent method is then employed to push the present best individual

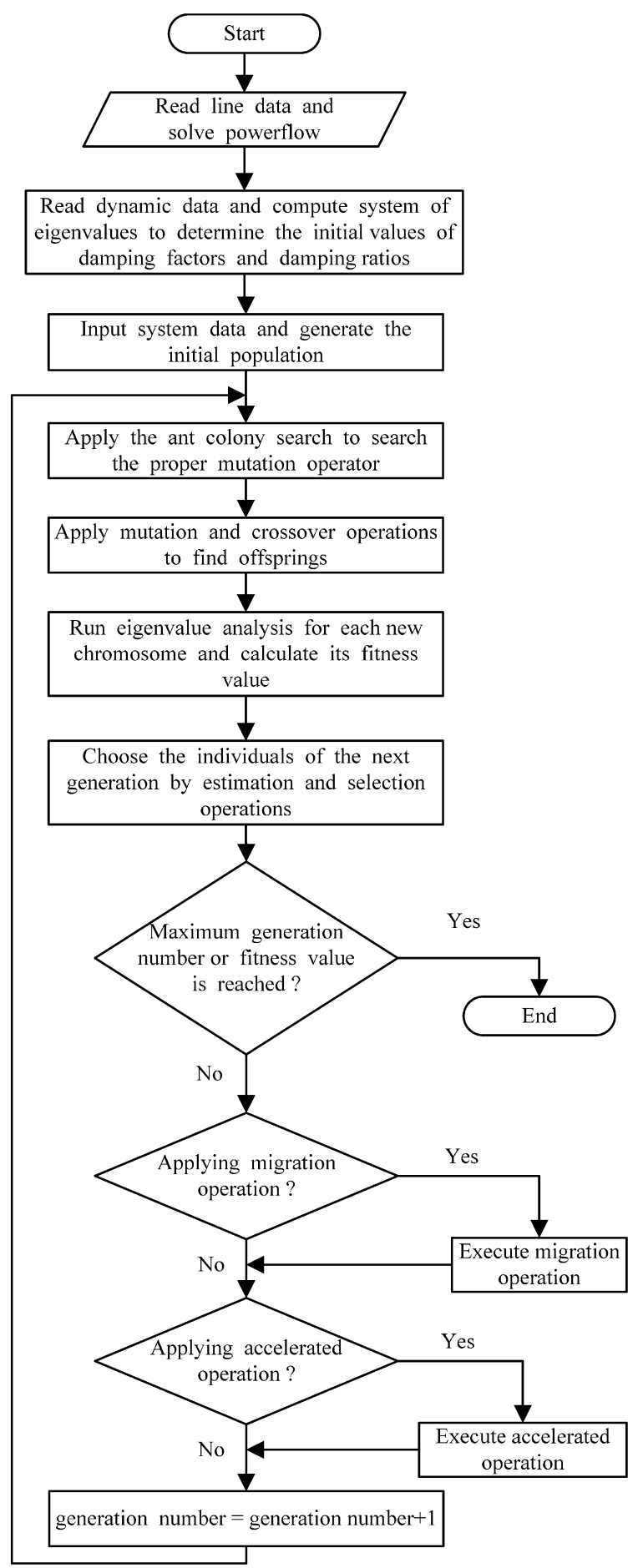

Fig. 1. Main calculation procedures of the proposed method. toward attaining a better point. Thus, the accelerated phase is expressed as follows.

$$
\left(\bar{I}_{b}^{G+1}, \bar{X}_{b}^{G+1}\right)=\left\{\begin{array}{c}
\left(I_{b}^{G+1}, X_{b}^{G+1}\right), \text { if } F\left(I_{b}^{G+1}, X_{b}^{G+1}\right)<F\left(I_{b}^{G}, X_{b}^{G}\right) \\
\left(I_{b}^{G+1}, X_{b}^{G+1}\right)-\left.\alpha \nabla F(I, X)\right|_{\substack{I=I_{b}^{G+1} \\
X=X_{b}^{G+1}}} \text { otherwise }
\end{array}\right.
$$

Where $\left(\bar{I}_{b}^{G+1}, \bar{X}_{b}^{G+1}\right)$ denotes the best individual, as obtained from Eq. (20). The gradient of the objective function, $\nabla F$, can be approximately calculated by finite difference. The step size in Eq. (24) is determined by the descent property. Initially, $\alpha$ is set to one to obtain the new individual.

Step 8

Steps 2 to 7 are repeated until the maximum iteration quantity or the desired fitness value is reached

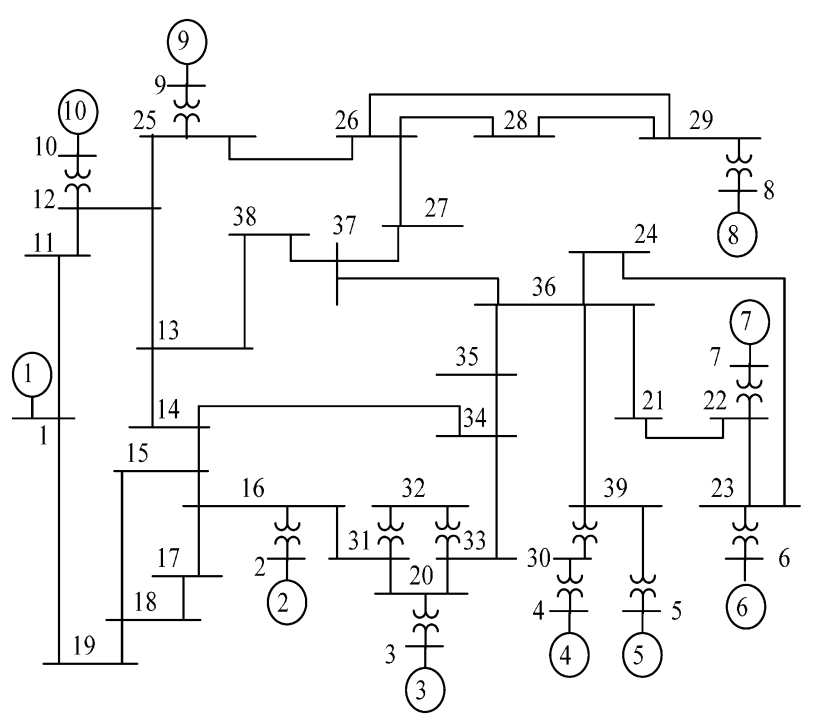

Fig. 2. New England 10-machine, 39-bus system.

Table 1

Searched gains and time constants of PSSs by the MIADHDE.

\begin{tabular}{clllllll}
\hline Objective function & Unit & $K_{S Z}$ & $T_{1 Z}$ & $T_{2 Z}$ & $T_{3 Z}$ & $T_{4 Z}$ & $\Delta \omega_{R Z}$ \\
\hline Objective function & G2 & 22.879 & 0.9478 & 1.0000 & 0.5638 & 0.0855 & - \\
with $F_{1}$ & G3 & 9.9292 & 1.1294 & 0.8259 & 1.4010 & 0.1363 & - \\
& G4 & 19.441 & 0.7037 & 0.0010 & 0.3081 & 1.0000 & - \\
& G5 & 4.6526 & 0.4137 & 0.0034 & 0.4088 & 0.2991 & - \\
& G6 & 25.983 & 0.8504 & 0.0680 & 0.6585 & 1.0000 & - \\
& G7 & 25.337 & 1.3027 & 0.4465 & 1.8283 & 0.5807 & - \\
& G8 & 12.609 & 1.3954 & 0.1049 & 0.5036 & 0.5714 & - \\
Objective function & G9 & 1.9189 & 1.4208 & 0.0357 & 1.2558 & 0.8001 & - \\
with F & G10 & 5.2901 & 0.0520 & 0.6492 & 0.0274 & 0.5501 & - \\
& G2 & 12.081 & 0.9993 & 0.8381 & 1.9811 & 0.1036 & - \\
& G3 & 25.806 & 0.9094 & 0.4710 & 0.5889 & 0.1607 & - \\
& G4 & 1.6731 & 1.1985 & 0.9523 & 1.6601 & 0.0514 & - \\
& G5 & 15.362 & 1.4677 & 0.1265 & 1.1015 & 0.9676 & - \\
& G6 & 2.5105 & 1.3828 & 0.0773 & 0.9510 & 0.2391 & - \\
& G7 & 27.314 & 0.7836 & 0.9998 & 1.5500 & 0.2323 & - \\
& G8 & 13.351 & 0.4623 & 0.0960 & 0.5360 & 0.2294 & - \\
& G9 & 40.825 & 0.9267 & 0.9697 & 0.3875 & 0.0248 & - \\
Objective function & G10 & 35.040 & 1.9999 & 0.0621 & 1.1086 & 0.6482 & - \\
with F $F_{3}$ & G2 & 5.6913 & 1.9998 & 0.0226 & 0.6646 & 0.5861 & - \\
& G3 & 5.3500 & 1.2156 & 0.7453 & 0.8590 & 0.0489 & $\Delta \omega_{8}$ \\
& G4 & 18.907 & 0.7508 & 0.9990 & 0.6400 & 0.1054 & $\Delta \omega_{8}$ \\
& G5 & 29.716 & 1.1765 & 0.1375 & 0.7536 & 0.6921 & - \\
& G6 & 20.367 & 0.1622 & 0.0167 & 0.7440 & 0.3808 & - \\
& G7 & 10.896 & 1.9996 & 0.1486 & 0.7937 & 0.9121 & - \\
& G8 & 39.123 & 0.7129 & 0.0117 & 0.1653 & 0.2576 & - \\
& G9 & 10.212 & 0.7475 & 0.1184 & 1.5247 & 0.6918 & $\Delta \omega_{2}$ \\
& G10 & 13.134 & 1.1229 & 0.0228 & 0.8460 & 0.4242 & $\Delta \omega_{7}$ \\
\hline
\end{tabular}


These computational procedures search configurations with various gain and time constants of PSS parameters so as to successively reduce the fitness function value. The solution procedures start off with performing calculations of power flows for various operating conditions, and then small signal analysis calculates the eigenvalues, oscillation frequencies, and damping ratios for various operating conditions of the complete system. This computational process of the MIADHDE algorithm for finding the optimal small signal stability solution is stated using the flowchart shown in Fig. 1.

\section{Simulation results}

The New England 10-machine, 39-bus system is used to illustrate the performance of the proposed method [35-36]. Fig. 2 presents the one-line diagram of the system. This system, which is unstable without PSS, has been widely used as a benchmark system for PSS parameter tuning problems. In the test system, except that the first bus is an infinite bus, other all generators are equipped with PSSs, and generator parameters are modified to add sub-transient parameters. There are four operating conditions considered as follows:

- Case 0: Base case.

- Case 1: The tie line $16-31$ is out-of-service.

- Case 2: Tie lines 14-34 and 36, 37 are out-of-service.

- Case 3: Lines 16-31 and 21, 22 are out-of-service, loads at buses 21 and 36 are increased by $30 \%$ and power generation of G1 is increased by $30 \%$.

In the tuning process, the constraints on PSS time constants $T_{1 N}$, $T_{2 N}, T_{3 N}$, and $T_{4 N}$ are set with the lower limit at $0.001 \mathrm{~s}$ and the upper limit at $2.0 \mathrm{~s}$, and the gain $K_{S N}$ of PSS ranges from 1 to 50 . There are 45 real variables and 9 integer variables to be optimized in this paper. Parameters $\sigma_{0}$ and $\zeta_{0}$ are set to be $-1.0 \%$ and $15 \%$, respectively. The target values of the maximum damping factor and the minimum damping ratio are assumed the desired limit value of all eigenvalues in the study system [9]. The weight $\varpi$

Table 2

Eigenvalues, oscillation frequencies, and damping ratios with and without PSSs for four cases.

\begin{tabular}{|c|c|c|c|c|}
\hline & Case 0 & Case 1 & Case 2 & Case 3 \\
\hline \multirow{9}{*}{ Without PSSs } & $-0.318 \pm 9.677 \mathrm{i}, 1.540,0.033$ & $-0.319 \pm 9.683 \mathrm{i}, 1.541,0.033$ & $-0.326 \pm 9.681 \mathrm{i}, 1.541,0.034$ & $-0.332 \pm 9.722 \mathrm{i}, 1.547,0.034$ \\
\hline & $-0.415 \pm 9.598 \mathrm{i}, 1.527,0.043$ & $-0.413 \pm 9.604 \mathrm{i}, 1.529,0.043$ & $-0.403 \pm 9.613 \mathrm{i}, 1.529,0.042$ & $-0.351 \pm 9.575 \mathrm{i}, 1.523,0.036$ \\
\hline & $-0.122 \pm 8.093 \mathrm{i}, 1.288,0.015$ & $-0.122 \pm 8.081 \mathrm{i}, 1.286,0.015$ & $-0.399 \pm 8.086 \mathrm{i}, 1.287,0.049$ & $-0.403 \pm 8.316 \mathrm{i}, 1.323,0.048$ \\
\hline & $-0.402 \pm 8.096 \mathrm{i}, 1.288,0.050$ & $-0.403 \pm 8.103 \mathrm{i}, 1.290,0.050$ & $-0.253 \pm 7.894 \mathrm{i}, 1.256,0.032$ & $-0.095 \pm 8.009 \mathrm{i}, 1.274,0.011$ \\
\hline & $-0.276 \pm 7.856 \mathrm{i}, 1.250,0.035$ & $-0.165 \pm 7.189 \mathrm{i}, 1.144,0.023$ & $-0.047 \pm 7.825 \mathrm{i}, 1.245,0.006$ & $\underline{0.002 \pm 7.139 i, 1.136,-0.001}$ \\
\hline & $-0.143 \pm 7.235 \mathrm{i}, 1.151,0.020$ & $-0.117 \pm 7.241 \mathrm{i}, 1.153,0.016$ & $-0.167 \pm 7.133 \mathrm{i}, 1.135,0.023$ & $-0.035 \pm 6.516 \mathrm{i}, 1.037,0.005$ \\
\hline & $-0.013 \pm 6.512 \mathrm{i}, 1.036,0.002$ & $0.003 \pm 6.479 \mathrm{i}, 1.031,-0.000$ & $0.010 \pm 6.550 \mathrm{i}, 1.042,-0.001$ & $\underline{\mathbf{0 . 1 5 8}} \pm 6.382 \mathrm{i}, 1.015,-0.025$ \\
\hline & $\mathbf{0 . 1 0 5} \pm 6.280 \mathrm{i}, 1.000,-0.017$ & $\mathbf{0 . 1 1 6} \pm 6.286 \mathrm{i}, 1.001,-0.018$ & $0.149 \pm 5.232 \mathrm{i}, 0.832,-0.029$ & $0.121 \pm 5.843 \mathrm{i}, 0.930,-0.021$ \\
\hline & $0.078 \pm 4.084 \mathrm{i}, 0.650,-0.019$ & $0.099 \pm 3.961 \mathrm{i}, 0.630,-0.025$ & $\mathbf{0 . 1 7 8} \pm 3.277 \mathrm{i}, 0.522,-0.054$ & $0.136 \pm 3.766 \mathrm{i}, 0.599,-0.036$ \\
\hline \multirow{4}{*}{ With PSSs } & $-2.179 \pm 13.18 \mathrm{i}, 2.098,0.163$ & $-2.175 \pm 13.15 \mathrm{i}, 2.094,0.163$ & $-2.210 \pm 13.23 \mathrm{i}, 2.106,0.165$ & $-2.166 \pm 13.13 \mathrm{i}, 2.090,0.163$ \\
\hline & $-2.586 \pm 11.30 \mathrm{i}, 1.798,0.223$ & $-2.605 \pm 11.26 \mathrm{i}, 1.793,0.225$ & $-2.257 \pm 10.63 \mathrm{i}, 1.692,0.208$ & $-2.893 \pm 9.793 \mathrm{i}, 1.559,0.283$ \\
\hline & $-2.134 \pm 10.73 \mathrm{i}, 1.708,0.195$ & $-2.938 \pm 9.962 \mathrm{i}, 1.586,0.283$ & $-2.423 \pm 10.41 \mathrm{i}, 1.657,0.227$ & $-2.091 \pm 9.595 \mathrm{i}, 1.527,0.213$ \\
\hline & $-2.949 \pm 9.968 \mathrm{i}, 1.587,0.284$ & $-2.187 \pm 9.730 \mathrm{i}, 1.549,0.219$ & $-2.936 \pm 9.901 \mathrm{i}, 1.576,0.284$ & $-1.603 \pm 9.351 \mathrm{i}, 1.488,0.169$ \\
\hline \multirow{5}{*}{$\begin{array}{c}\text { Objective } \\
\text { function with } F_{1}\end{array}$} & $-1.643 \pm 9.180 \mathrm{i}, 1.461,0.176$ & $-1.602 \pm 9.198 \mathrm{i}, 1.464,0.172$ & $-1.446 \pm 9.436 \mathrm{i}, 1.502,0.151$ & $-2.766 \pm 8.865 \mathrm{i}, 1.411,0.298$ \\
\hline & $-1.461 \pm 8.772 \mathrm{i}, 1.396,0.164$ & $-1.420 \pm 8.768 \mathrm{i}, 1.395,0.160$ & $-1.933 \pm 8.646 \mathrm{i}, 1.376,0.218$ & $-1.355 \pm 8.661 \mathrm{i}, 1.378,0.155$ \\
\hline & $-1.822 \pm 8.636 \mathrm{i}, 1.375,0.206$ & $-1.829 \pm 8.563 \mathrm{i}, 1.363,0.209$ & $-1.429 \pm 7.927 \mathrm{i}, 1.262,0.177$ & $-1.705 \pm 8.397 \mathrm{i}, 1.337,0.199$ \\
\hline & $\mathbf{- 1 . 1 7 6} \pm 7.314 \mathrm{i}, 1.164,0.159$ & $\mathbf{- 1 . 1 7 0} \pm 7.308 \mathrm{i}, 1.163,0.157$ & $\mathbf{- 1 . 2 8 3} \pm 6.740 \mathrm{i}, 1.073,0.187$ & $\mathbf{- 1 . 1 8 2} \pm 7.631 \mathrm{i}, 1.215,0.153$ \\
\hline & $-2.075 \pm 4.790 \mathrm{i}, 0.762,0.397$ & $-2.238 \pm 4.848 \mathrm{i}, 0.771,0.419$ & $-2.898 \pm 4.554 \mathrm{i}, 0.725,0.537$ & $-2.355 \pm 5.170 \mathrm{i}, 0.822,0.415$ \\
\hline \multirow{4}{*}{ With PSSs } & $-3.931 \pm 13.55 \mathrm{i}, 2.156,0.279$ & $-3.932 \pm 13.51 \mathrm{i}, 2.151,0.279$ & $-3.789 \pm 13.29 \mathrm{i}, 2.115,0.274$ & $-3.989 \pm 13.19 \mathrm{i}, 2.098,0.290$ \\
\hline & $-2.599 \pm 12.27 \mathrm{i}, 1.952,0.207$ & $-4.787 \pm 11.23 \mathrm{i}, 1.788,0.392$ & $-2.675 \pm 11.95 \mathrm{i}, 1.901,0.219$ & $-3.050 \pm 11.89 \mathrm{i}, 1.892,0$. \\
\hline & $-4.766 \pm 11.24 \mathrm{i}, 1.789,0.390$ & $-3.202 \pm 11.75 \mathrm{i}, 1.870,0.263$ & $-3.216 \pm 11.65 \mathrm{i}, 1.853,0.266$ & $-2.802 \pm 11.35 \mathrm{i}, 1.806,0.240$ \\
\hline & $-2.856 \pm 11.65 \mathrm{i}, 1.854,0.238$ & $-2.417 \pm 11.46 \mathrm{i}, 1.823,0.206$ & $\mathbf{- 2 . 0 2 0} \pm 11.44 i, 1.821,0.174$ & $-4.110 \pm 10.37 \mathrm{i}, 1.649,0.369$ \\
\hline \multirow{5}{*}{$\begin{array}{c}\text { Objective } \\
\text { function with } \mathrm{F}_{2}\end{array}$} & $-2.089 \pm 11.43 \mathrm{i}, 1.818,0.180$ & $\mathbf{- 1 . 8 7 1} \pm 11.03 \mathrm{i}, 1.755,0.167$ & $-4.101 \pm 10.93 \mathrm{i}, 1.740,0.351$ & $-2.124 \pm 11.00 \mathrm{i}, 1.750,0.190$ \\
\hline & $-\mathbf{1 . 9 1 8} \pm 10.91 \mathrm{i}, 1.736,0.173$ & $-2.071 \pm 10.92 \mathrm{i}, 1.737,0.186$ & $-2.274 \pm 10.86 \mathrm{i}, 1.728,0.205$ & $-2.130 \pm 10.73 \mathrm{i}, 1.706,0.195$ \\
\hline & $-3.750 \pm 10.35 \mathrm{i}, 1.646,0.341$ & $-3.703 \pm 10.31 \mathrm{i}, 1.640,0.338$ & $-3.258 \pm 9.764 \mathrm{i}, 1.554,0.317$ & $-4.265 \pm 8.789 \mathrm{i}, 1.398,0.437$ \\
\hline & $-2.236 \pm 7.368 \mathrm{i}, 1.172,0.290$ & $-2.244 \pm 7.378 \mathrm{i}, 1.174,0.291$ & $-3.345 \pm 7.077 \mathrm{i}, 1.126,0.427$ & $\mathbf{- 1 . 8 7 4} \pm 7.283 \mathrm{i}, 1.159,0.249$ \\
\hline & $-3.127 \pm 4.435 \mathrm{i}, 0.706,0.576$ & $-3.162 \pm 4.419 \mathrm{i}, 0.703,0.582$ & $-3.115 \pm 4.455 \mathrm{i}, 0.709,0.573$ & $-3.112 \pm 4.527 \mathrm{i}, 0.720,0.567$ \\
\hline \multirow{4}{*}{ With PSSs } & $-2.327 \pm 13.10 \mathrm{i}, 2.085,0.175$ & $-2.310 \pm 13.11 \mathrm{i}, 2.086,0.174$ & $-2.249 \pm 12.99 \mathrm{i}, 2.068,0.171$ & $-2.178 \pm 12.94 \mathrm{i}, 2.060,0.165$ \\
\hline & $-2.051 \pm 12.79 \mathrm{i}, 2.035,0.158$ & $-2.050 \pm 12.77 \mathrm{i}, 2.033,0.158$ & $-\mathbf{2 . 0 2 2} \pm 12.65 \mathrm{i}, 2.013,0.158$ & $-1.990 \pm 12.35 \mathrm{i}, 1.966,0.159$ \\
\hline & $-\mathbf{1 . 9 8 0} \pm 11.19 \mathrm{i}, 1.781,0.174$ & $-\mathbf{1 . 9 6 9} \pm 11.18 \mathrm{i}, 1.779,0.173$ & $-2.044 \pm 11.15 \mathrm{i}, 1.775,0.180$ & $-2.022 \pm 11.02 \mathrm{i}, 1.754,0.180$ \\
\hline & $-9.241 \pm 6.633 \mathrm{i}, 1.055,0.812$ & $-9.300 \pm 6.657 \mathrm{i}, 1.059,0.813$ & $-9.211 \pm 6.551 \mathrm{i}, 1.043,0.815$ & $-8.524 \pm 6.531 \mathrm{i}, 1.039,0.794$ \\
\hline \multirow{5}{*}{$\begin{array}{c}\text { Objective } \\
\text { function with } \mathrm{F}_{3}\end{array}$} & $-2.054 \pm 9.703 \mathrm{i}, 1.544,0.207$ & $-2.060 \pm 9.691 \mathrm{i}, 1.542,0.208$ & $-2.116 \pm 9.674 \mathrm{i}, 1.539,0.214$ & $\mathbf{- 1 . 9 8 7} \pm 9.143 \mathrm{i}, 1.455,0.212$ \\
\hline & $-2.151 \pm 9.040 \mathrm{i}, 1.438,0.231$ & $-2.166 \pm 8.854 \mathrm{i}, 1.409,0.238$ & $-4.085 \pm 8.039 \mathrm{i}, 1.279,0.453$ & $-1.990 \pm 8.003 \mathrm{i}, 1.274,0.241$ \\
\hline & $-3.628 \pm 7.647 \mathrm{i}, 1.217,0.429$ & $-2.053 \pm 7.382 \mathrm{i}, 1.175,0.268$ & $-2.121 \pm 8.414 \mathrm{i}, 1.339,0.244$ & $-3.797 \pm 7.088 \mathrm{i}, 1.128,0.472$ \\
\hline & $-2.644 \pm 7.434 \mathrm{i}, 1.183,0.335$ & $-3.782 \pm 6.817 \mathrm{i}, 1.085,0.485$ & $-2.478 \pm 6.318 \mathrm{i}, 1.005,0.365$ & $-2.164 \pm 6.371 \mathrm{i}, 1.014,0.322$ \\
\hline & $-2.221 \pm 4.751 \mathrm{i}, 0.756,0.423$ & $-2.007 \pm 4.497 \mathrm{i}, 0.716,0.407$ & $-3.113 \pm 5.124 \mathrm{i}, 0.815,0.519$ & $-2.525 \pm 4.066 \mathrm{i}, 0.647,0.527$ \\
\hline \multirow{9}{*}{$\begin{array}{l}\text { With above } \\
\text { parameters of } \\
\text { PSSs, but lost } \\
\text { remote signal at } \\
\text { input of PSSs }\end{array}$} & $-2.242 \pm 13.04 \mathrm{i}, 2.075,0.169$ & $-2.239 \pm 13.03 \mathrm{i}, 2.074,0.169$ & $-2.151 \pm 12.91 \mathrm{i}, 2.054,0.164$ & $-2.212 \pm 12.90 \mathrm{i}, 2.054,0.169$ \\
\hline & $-2.012 \pm 12.74 \mathrm{i}, 2.028,0.156$ & $-2.010 \pm 12.73 \mathrm{i}, 2.026,0.156$ & $-2.035 \pm 12.64 \mathrm{i}, 2.012,0.159$ & $-1.966 \pm 12.32 \mathrm{i}, 1.961,0.158$ \\
\hline & $-2.008 \pm 11.22 \mathrm{i}, 1.787,0.176$ & $-1.999 \pm 11.22 \mathrm{i}, 1.786,0.175$ & $\mathbf{- 2 . 0 1 6} \pm 11.21 \mathrm{i}, 1.784,0.177$ & $-2.028 \pm 11.10 \mathrm{i}, 1.768,0.180$ \\
\hline & $-2.067 \pm 9.737 \mathrm{i}, 1.549,0.208$ & $-2.063 \pm 9.720 \mathrm{i}, 1.547,0.208$ & $-2.080 \pm 9.694 \mathrm{i}, 1.543,0.210$ & $-1.912 \pm 9.117 \mathrm{i}, 1.451,0.205$ \\
\hline & $-8.828 \pm 6.001 \mathrm{i}, 0.955,0.827$ & $-8.745 \pm 6.001 \mathrm{i}, 0.955,0.825$ & $-8.427 \pm 5.404 \mathrm{i}, 0.860,0.842$ & $-8.093 \pm 5.908 \mathrm{i}, 0.940,0.808$ \\
\hline & $-\mathbf{- 1 . 7 2 1} \pm 8.807 \mathrm{i}, 1.401,0.192$ & $\mathbf{- 1 . 7 1 4} \pm 8.772 \mathrm{i}, 1.396,0.192$ & $-2.140 \pm 8.137 \mathrm{i}, 1.295,0.254$ & $\mathbf{- 1 . 8 9 8} \pm 8.302 \mathrm{i}, 1.321,0.223$ \\
\hline & $-2.483 \pm 7.984 \mathrm{i}, 1.270,0.297$ & $-2.168 \pm 7.544 \mathrm{i}, 1.201,0.276$ & $-2.981 \pm 7.928 \mathrm{i}, 1.262,0.352$ & $-2.876 \pm 6.698 \mathrm{i}, 1.066,0.395$ \\
\hline & $-3.550 \pm 7.517 \mathrm{i}, 1.196,0.427$ & $-3.375 \pm 6.884 \mathrm{i}, 1.096,0.440$ & $-2.786 \pm 6.774 \mathrm{i}, 1.078,0.380$ & $-1.969 \pm 6.661 \mathrm{i}, 1.060,0.283$ \\
\hline & $-2.228 \pm 3.758 \mathrm{i}, 0.598,0.510$ & $-2.065 \pm 3.470 \mathrm{i}, 0.552,0.511$ & $-3.243 \pm 3.863 \mathrm{i}, 0.615,0.643$ & $-3.255 \pm 3.158 \mathrm{i}, 0.503,0.718$ \\
\hline
\end{tabular}


parameter is set to be 1.35 , which is derived from the experiences of many experiments conducted on this problem. Also, in the MIADHDE algorithm, there are nine parameters to be set up first before carrying out the optimization process. The first seven parameters include population size $N_{P}$, crossover factor $C r$, maximum iterations itermax, tolerance of gene diversity, $\alpha, \beta, Q$ set to be $N_{P}=5, C r=0.6$, itermax $=5000$, tolerance of gene diversity $=0$ for integer variable, and tolerance of gene diversity $=0.1$ for real variables, $\alpha=2, \beta=1$, and $Q=1$. The other two parameters, scaling factor $\kappa$ and tolerance of population diversity $\varepsilon_{1}$, are relatively sensitive in this optimization process. Therefore, the two parameters are set according to the experiences of many experiments on the problem of tuning PSS parameters. The best values of parameters $\kappa$ and $\varepsilon_{1}$ are 0.3 and 0.1 , respectively. The study system using three different objective functions are performed 20 times by the MIADHDE algorithm, and then the best solution is presented. The software was written in Matlab and executed on a Pentium $42.0 \mathrm{GHz}$ with 512MB of RAM. The average CPU time for with and without remote signals are 71267 and 10271 seconds, respectively.

Table 1 presents the PSS parameters searched by the MIADHDE method. Table 2 presents the principal eigenvalues, oscillation frequencies, and damping ratios. In Table 2 , the boldface value represents the biggest damping factor, and the value enclosed in a square frame represents the smallest damping ratio. All damping factors are smaller than -1.0 and all damping ratios are greater than 0.15 in Table 2 . The results of the objective function $F_{2}$ show the minimum damping ratio and the maximum damping factor under all cases, which is better than the results of objective function $F_{1}$. Also, the results of $F_{3}$ are better than those of $F_{2}$. It means that the addition of remote feedback signals can increase the system dynamic stability. The principal eigenvalues are drawn in the s-planes, which are shown in Figs. 3-5, respectively. In Fig. 5, the scale of the damp factor axis is twice as large as in Figs. 3 and 4 . The results reveal that the proposed MIADHDE can not only relocate the unstable or lightly damped oscillation modes but also shift other oscillation modes more to the left in the s-plane. However, if the remote feedback signals are lost, then the system stability is slightly degraded. Clearly, the system performance obtained using the objective function $F_{3}$ is better than both objective functions $F_{1}$ and $F_{2}$.

A number of time domain simulations were performed to demonstrate the effectiveness of tuning parameters of PSSs using the proposed MIADHDE method. In these tests, a four-cycle three-phase fault is applied at bus 29. From Figs. 6-9, line powers from bus 29 to bus 26 are shown for illustration. The objective function $F_{1}$ is similar to best objective function in the literature [8], which is sustained oscillation over six seconds for any system operation case in Figs. 6-9. However, the objective function $F_{3}$ achieves a steady state the fastest of the three objective functions under any system operation conditions. These time domain simulations agree well with the results of eigenvalue analysis.

\section{Conclusions}

A novel mixed-integer ant direction hybrid differential evolution (MIADHDE) algorithm has been proposed to determine the optimal tuning of power system stabilizers with three different objective functions. The MIADHDE algorithm searches for the optimal PSS parameters, which can include remote feedback of speed deviation measurement signals. Eigenvalue analysis and nonlinear time domain simulation results demonstrate the effectiveness of the proposed algorithm. We believe the MIADHDE can serve as a better alternative to the problem of optimal tuning of power system stabilizers using the stochastic optimization approach.

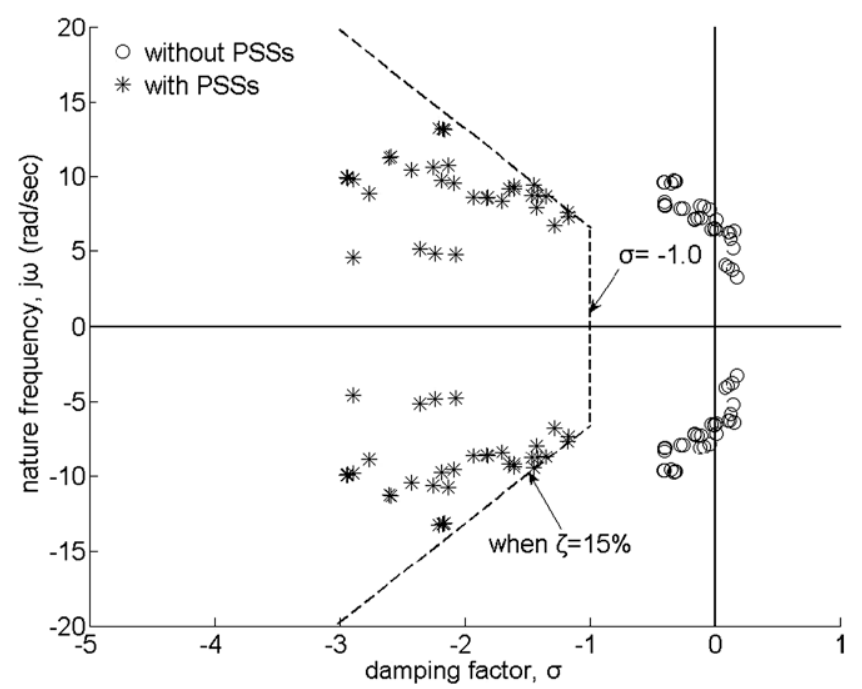

Fig. 3. The searched eigenvalues with objective function $F_{1}$ for four cases.

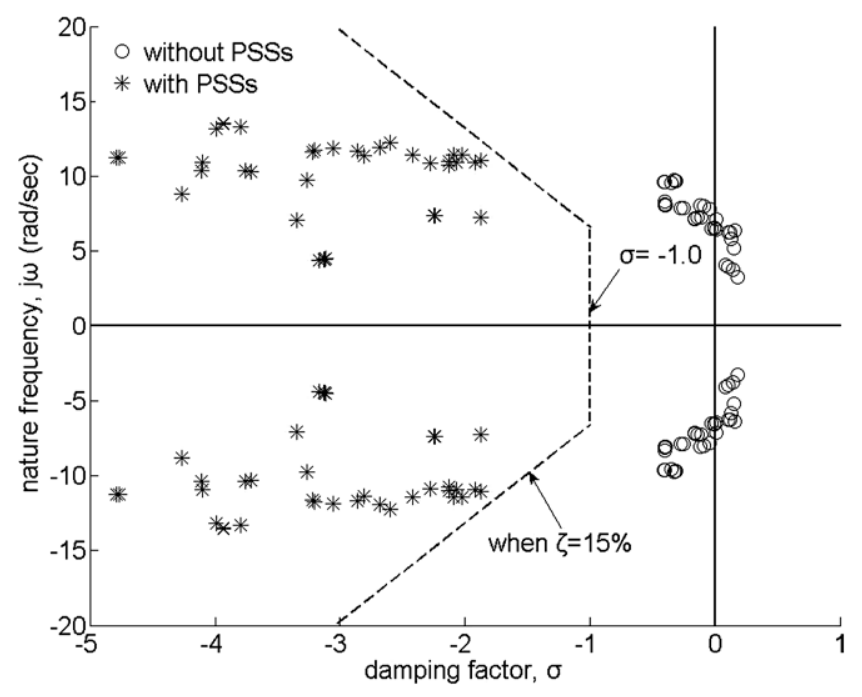

Fig. 4. The searched eigenvalues with objective function $F_{2}$ for four cases.

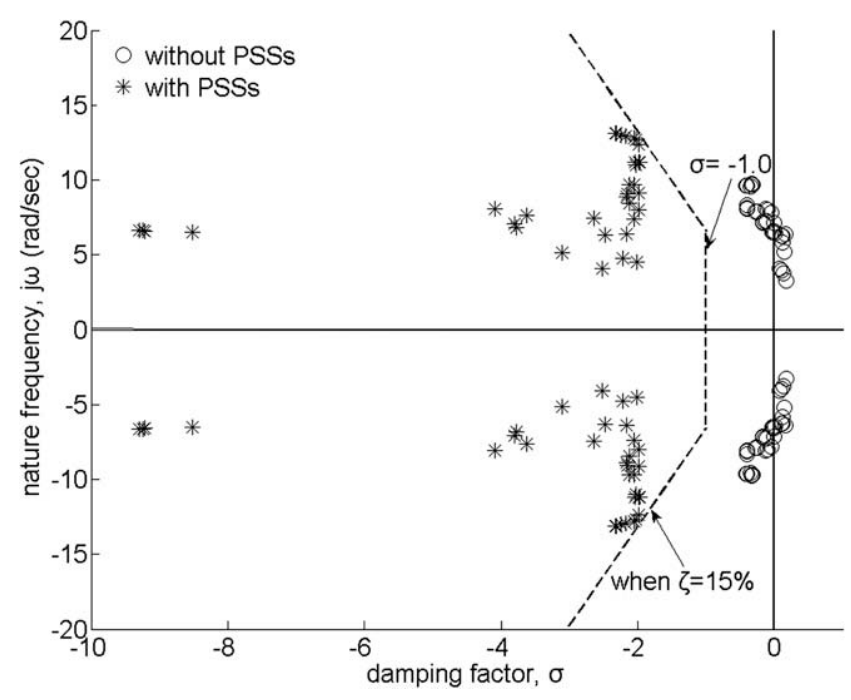

Fig. 5. The searched eigenvalues with objective function $F_{3}$ for four cases. 


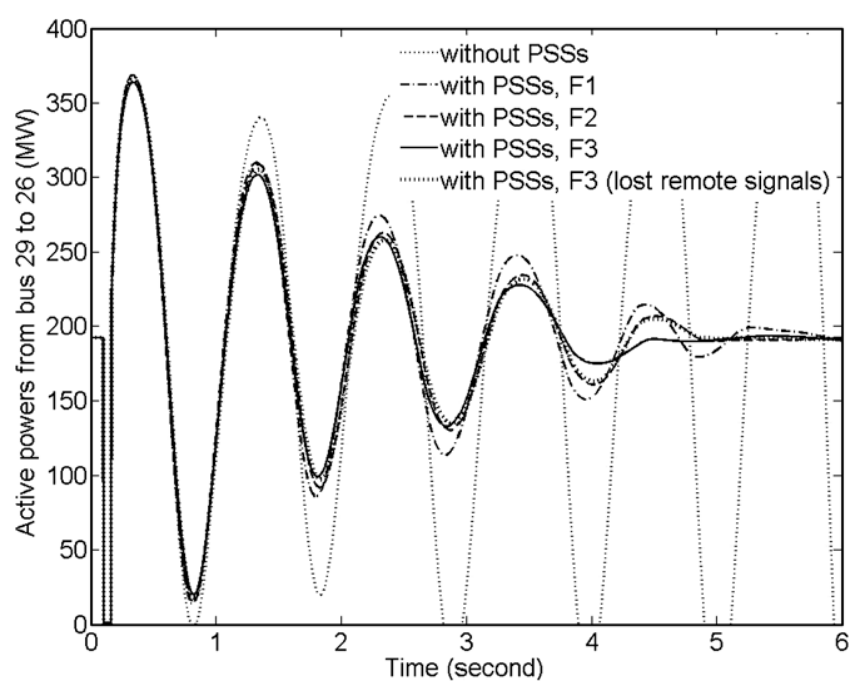

Fig. 6. The line powers from bus 29 to 26 for case 0 .

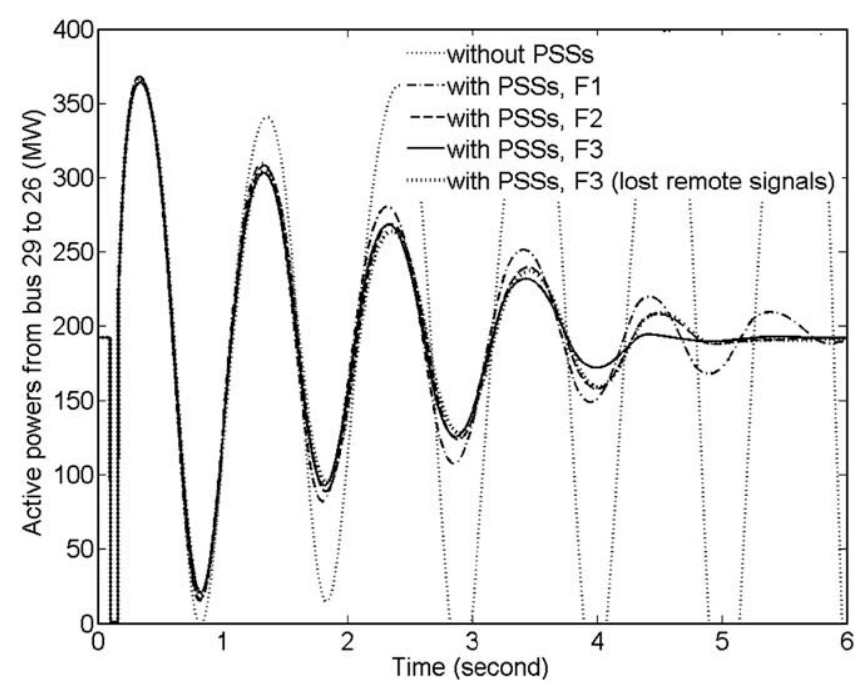

Fig. 7. The line powers from bus 29 to 26 for case 1 .

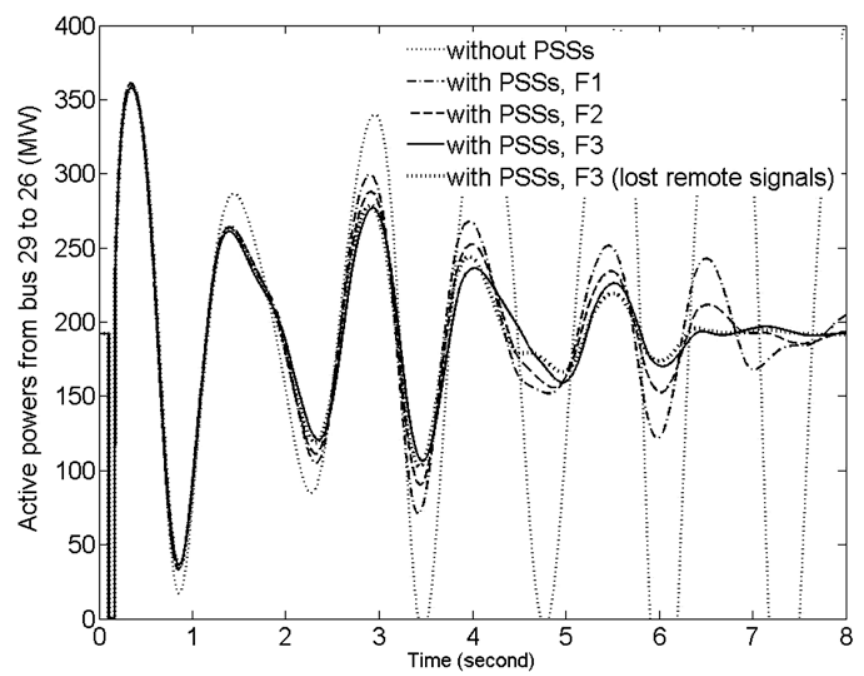

Fig. 8. The line powers from bus 29 to 26 for case2.

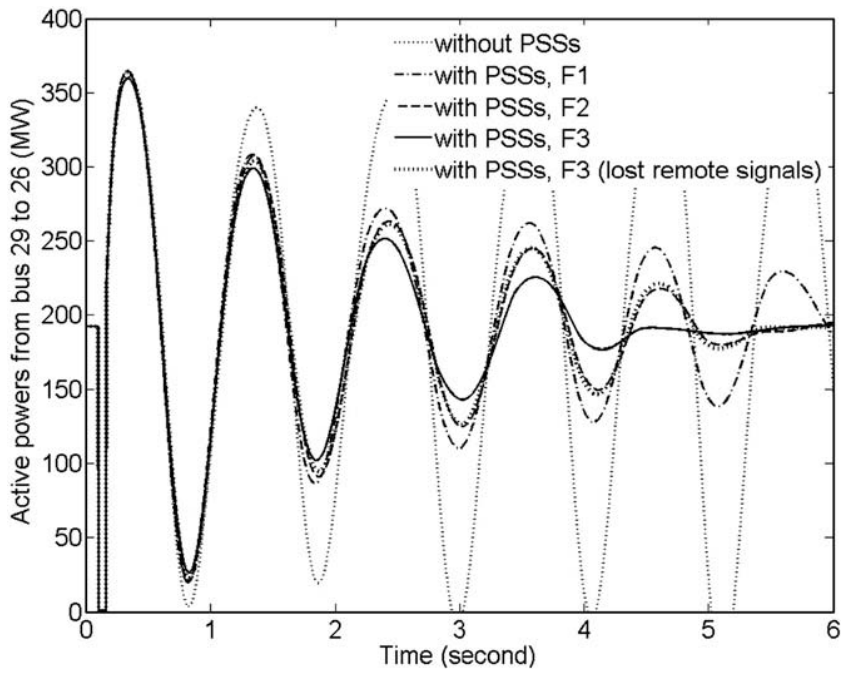

Fig. 9. The line powers from bus 29 to 26 for case3.

\section{Acknowledgment}

The authors would like to express their sincere gratitude to financial support of this work by the National Science Council of the ROC under contract number NSC-97-2221-E-131-024.

\section{References}

[1] Messina AR, Ramirez JM, Canedo C. An investigation on the use of power system stabilizers for damping inter-area oscillations in longitudinal power systems. IEEE Trans Power Syst 1998;13(2):552-9.

[2] Liu C, Yokoyama R, Koyanagi K, Lee KY. PSS design for damping of inter-area power oscillations by coherency-based equivalent model. Int J Elec Power 2004;26(7):535-44.

[3] Klein M, Rgers GJ, Moorty S, Kundur P. Analytical investigation of factors influencing power system stabilizers performance. IEEE Trans Energy Conver 1992:7(3):382-90.

[4] Kundur P, Klein M, Rogers GJ, Zywno MS. Application of power system stabilizers for enhancement of overall system stability. IEEE Trans Power Syst 1989;4(2):614-26.

[5] Klein M, Rogers GJ, Kundur P. A fundamental study of inter-area oscillations in power systems. IEEE Trans Power Syst 1991;6(3):914-21.

[6] Kundur P. Effective use of power system stabilizers for enhancement of power system reliability. IEEE Power Eng Soc Summer Meeting 1999;1:96-103.

[7] Ishimaru M, Yokoyama R, Neto OM, Lee KY. Allocation and design of power system stabilizers for mitigating low-frequency oscillations in the eastern interconnected power system in Japan. Int J Elec Power 2004;26(8):607-18.

[8] Abdel-Magid YL, Abido MA. Optimal multi objective design of robust power system stabilizers using genetic algorithms. IEEE Trans Power Syst 2003;18(3):1125-32.

[9] Hongesombut K, Mitani Y, Dechanupaprittha S, Ngamroo I, Pasupa K, Tippayachai J. Power system stabilizer tuning based on multiobjective design using hierarchical and parallel micro genetic algorithm. IEEE Int Conf Power Syst Technol 2004;1:402-7.

[10] Abido MA. A novel approach to conventional power system stabilizer design using tabu search. Int J Elec Power 1999;21(6):443-54.

[11] Abido MA. Robust design of multi-machine power system stabilizers using simulated annealing. IEEE Trans Energy Conver 2000;15(3):297-304.

[12] Al-Awami AT, Abdel-Magid YL, Abido MA. A particle-swarm-based approach of power system stability enhancement with unified power flow controller. Int J Elec Power 2007;29(3):251-9.

[13] Abido MA, Abdel-Magid YL. Optimal design of power system stabilizers using evolutionary programming. IEEE Trans Energy Conver 2002;17(4):429-36.

[14] Do Bomfim ALB, Taranto GN, Falcao DM. Simultaneous tuning of power system damping controllers using genetic algorithms. IEEE Trans Power Syst 2000;15(1):163-9.

[15] Phadke AG. Synchronized phasor measurements a historical overview. IEEE PES Conf Exhibition Trans Distrib 2002:1:476-9.

[16] Dou C-X, Jia Q-Q, Jin S-J, Bo Z-Q. Delay-independent decentralized stabilizer design for large interconnected power systems based on WAMS. Int J Elec Power 2007;29(10):775-82.

[17] Snyder AF, Hadjsaid N, Georges D, Mili L, Phadke AG, Faucon O, et al. Inter-area oscillation damping with power system stabilizers and synchronized phasor measurements. IEEE Proc Int Conf Power Syst Technol 1998;2:790-4. 
[18] Kamwa I, Gerin-Lajoie L, Trudel G. Multi-loop power system stabilizers using wide-area synchronous phasor measurements. Proc Am Control Conf 1998;5:2963-7.

[19] Kamwa I, Grondin R, Hebert Y. Wide-area measurement based stabilizing control of large power systems a decentralized/hierarchical approach. IEEE Trans Power Syst 2001;16(1):136-53.

[20] Hasanović A, Feliachi A. Genetic algorithm based inter-area oscillation damping controller design using MATLAB. IEEE Power Eng Soc Summer Meeting 2002;3:1136-41.

[21] Su C-T, Lee C-S. Modified differential evolution method for capacitor placement of distribution systems. IEEE/PES Trans Distrib Conf Exhibition 2002;1:208-13.

[22] Su C-T, Lee C-S. Network reconfiguration of distribution systems using improved mixed-integer hybrid differential evolution. IEEE Trans Power Deliver 2003;18(3):1022-7.

[23] Chiou JP, Chang CF, Su CT. Ant direction hybrid differential evolution for solving large capacitor placement problems. IEEE Trans Power Syst 2004;19(4):1794-800.

[24] Chiou JP, Wang FS. Hybrid method of evolutionary algorithms for static and dynamic optimization problems with application to a fed-batch fermentation process. J Comput Chem Eng 1999;23:1277-91.

[25] Chiou JP, Wang FS. Estimation of monod model parameters by hybrid differential evolution. J Bioprocess Biosyst Eng 2001;1:109-13.

[26] Kundur P. Power system stability and control. New York: McGraw-Hill; 1993.
[27] Haque MH, Rahim AHMA. Determination of first swing stability limit of multimachine power systems through Taylor series expansions. IEE Proc C 1989;136(6):373-80.

[28] Colorni A, Dorigo M, Maniezzo V. An investigation of some properties of an ant algorithm. In: Proc. conf. parallel problem solving from nature, vol. 1 Amsterdam: North-Holland; 1992 p. 509-20.

[29] Dorigo M, Gambardella LM. Ant colonies for the traveling salesman problem. BioSystems; 1997.

[30] Yu IK, Chou CS, Song YH. Application of the ant colony search algorithm to short-term generation scheduling problem of thermal units. Proc Int Conf Power Syst Technol 1998;1:552-6.

[31] Sisworahardio NS, El-Keib AA. Unit commitment using the ant colony search algorithm. Conf Power Eng 2002 Large Eng Syst 2002:2-6.

[32] Huang SJ. Enhancement of hydroelectric generation scheduling using ant colony system based optimization approaches. IEEE Trans Energy Conver 2001;16(3):296-301.

[33] Storn R, Price K. Minimizing the real functions of the ICEC '96 contest by differential evolution. IEEE Proc Int Conf Evolut Comput 1996:842-4 [Nagoya, Japan].

[34] Storn R. On the usage of differential evolution for function optimization. NAFIPS 1996. Biennial Conf of the North American 1996: 519-23 [Berkeley].

[35] Pai MA. Energy function analysis for power system stability. Norwell: Kluwer; 1989.

[36] Padiyar KR. Power system dynamics stability and control. New York: John Wiley; 1996. 